\title{
ON PRODUCTS OF IDEMPOTENT MATRICES
}

\author{
by J. A. ERDOS
}

(Received 5 August, 1966)

In [1], J. M. Howie considered the semigroup of transformations of sets and proved (Theorem 1) that every transformation of a finite set which is not a permutation can be written as a product of idempotents. In view of the analogy between the theories of transformations of finite sets and linear transformations of finite dimensional vector spaces, Howie's theorem suggests a corresponding result for matrices. The purpose of this note is to prove such a result.

THEOREM. Every singular square matrix can be written as a product of idempotent matrices.

Proof. If $E$ is an idempotent matrix and $P$ is non-singular, then $P^{-1} E P$ is also idempotent and hence it is sufficient to prove that every singular matrix is similar to a product of idempotents. Two separate proofs of this are given. The first is an induction proof for matrices that are similar to triangular matrices and hence holds in general only for matrices over an algebraically closed field. For a proof valid for matrices over any field an explicit construction is given in II. It is clear that II is sufficient to establish the result but as it relies on the "rational canonical form " and as I is so simple, it is of interest to include both proofs.

I. Proof for matrices that are similar to triangular matrices.

We proceed by induction on the order of the matrix, and make the induction hypothesis that the theorem is valid for such matrices of order $n-1$. Suppose that a matrix $A$ is of order $n$. As $A$ is singular it is similar to an upper triangular matrix whose first column is zero and so we may partition this matrix as

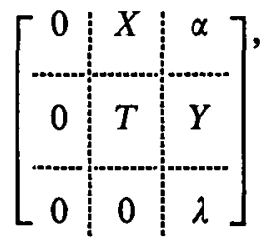

where $T$ is of order $n-2$. If $T$ is singular, so is

and by the induction hypothesis,

$$
\left[\begin{array}{ll}
T & Y \\
0 & \lambda
\end{array}\right]
$$

$$
\left[\begin{array}{lll}
1 & 0 & 0 \\
0 & T & Y \\
0 & 0 & \lambda
\end{array}\right]
$$


can be written as a product of idempotents. But

$$
\left[\begin{array}{ccc}
1 & 0 & 0 \\
0 & T & Y \\
0 & 0 & \lambda
\end{array}\right]\left[\begin{array}{lll}
0 & X & \alpha \\
0 & I & 0 \\
0 & 0 & 1
\end{array}\right]=\left[\begin{array}{ccc}
0 & X & \alpha \\
0 & T & Y \\
0 & 0 & \lambda
\end{array}\right]
$$

and the postmultiplying matrix is idempotent. So it is sufficient to consider the case when $T$ is non-singular.

Let $Z=X T^{-1}$, and let

$$
B=\left[\begin{array}{lll}
0 & X & 0 \\
0 & T & 0 \\
0 & 0 & 1
\end{array}\right]
$$

Then by the induction hypothesis $B$ can be written as a product of idempotents. We now have two cases.

(i) $Z Y=\alpha$. Let

$$
\begin{aligned}
& E=\left[\begin{array}{ccc}
0 & Z & 0 \\
0 & I & 0 \\
1 & -Z & 1
\end{array}\right], \\
& F=\left[\begin{array}{ccc}
0 & Z & \lambda+\alpha \\
0 & I & Y \\
0 & 0 & 0
\end{array}\right] .
\end{aligned}
$$

Then a direct calculation shows that $E$ and $F$ are idempotents and, using $X=Z T$, that

$$
E F B=A \text {. }
$$

(ii) $Z Y-\alpha=k \neq 0$. Let

$$
\begin{aligned}
E & =\left[\begin{array}{ccc}
1 & 0 & 0 \\
0 & I & 0 \\
-\lambda / k & (\lambda / k) Z & 0
\end{array}\right], \\
F & =\left[\begin{array}{ccc}
1 & 0 & \alpha \\
0 & I & Y \\
0 & 0 & 0
\end{array}\right]
\end{aligned}
$$

Again $E$ and $F$ are idempotents and

$$
E F B=A
$$


For the order 2 case, we have

if $\alpha \neq 0$ and

$$
\left[\begin{array}{ll}
0 & \alpha \\
0 & \lambda
\end{array}\right]=\left[\begin{array}{cc}
1 & 0 \\
\lambda / \alpha & 0
\end{array}\right]\left[\begin{array}{ll}
1 & \alpha \\
0 & 0
\end{array}\right]\left[\begin{array}{ll}
0 & 0 \\
0 & 1
\end{array}\right]
$$

$$
\left[\begin{array}{ll}
0 & 0 \\
0 & \lambda
\end{array}\right]=\left[\begin{array}{ll}
0 & 0 \\
1 & 1
\end{array}\right]\left[\begin{array}{ll}
1 & \lambda \\
0 & 0
\end{array}\right]\left[\begin{array}{ll}
0 & 0 \\
0 & 1
\end{array}\right]
$$

so the theorem is valid for matrices of order 2 .

The result follows.

II. Proof for the general case.

It is well known (see for example [2], Section III), that any matrix is similar to a matrix of the form

$$
A=\operatorname{diag}\left\{C_{1}, C_{2}, \ldots, C_{k}\right\},
$$

where $C_{i}$ is a square matrix of order $n_{i}$ of the form

$$
\left[\begin{array}{ccccc}
0 & 1 & 0 & \ldots & 0 \\
0 & 0 & 1 & \ldots & 0 \\
\vdots & & & & \\
0 & 0 & 0 & \ldots & 1 \\
a_{i} & b_{i} & c_{i} & \ldots & z_{i}
\end{array}\right]
$$

As $A$ is singular, we can arrange that $C_{k}$ is singular and then $a_{k}=0$. Note that if $n_{i}=1$, $C_{i}$ reduces to $\left[a_{i}\right]$. This reduction can be carried out over any field.

Let $E_{0}$ be the matrix

and, for $1 \leqq i \leqq n-1$, let $E_{i}$ be

$$
\left[\begin{array}{cc}
0 & 0 \\
0 & I_{n-1}
\end{array}\right]
$$

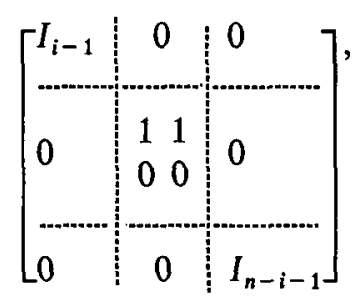

where $I_{i}$ denotes the identity matrix of order $t$. Then premultiplication of any matrix by $E_{i}$ adds the $(i+1)$ th row to the $i$ th row and reduces the $(i+1)$ th row to zero. $E_{i}$ is clearly idempotent. Let

$$
N=E_{n-1} E_{n-2} \ldots E_{1} E_{0}
$$


Then it is easily seen that

$$
N=\left[\begin{array}{ccccc}
0 & 1 & 0 & \ldots & 0 \\
0 & 0 & 1 & \ldots & 0 \\
0 & 0 & 0 & \ldots & 0 \\
\vdots & \vdots & \vdots & & \\
0 & 0 & 0 & \ldots & 1 \\
0 & 0 & 0 & \ldots & 0
\end{array}\right]
$$

For $i \leqq k$, let $r_{i}=\sum_{j=1}^{i-1} n_{j}$ and, for $i<k$, let

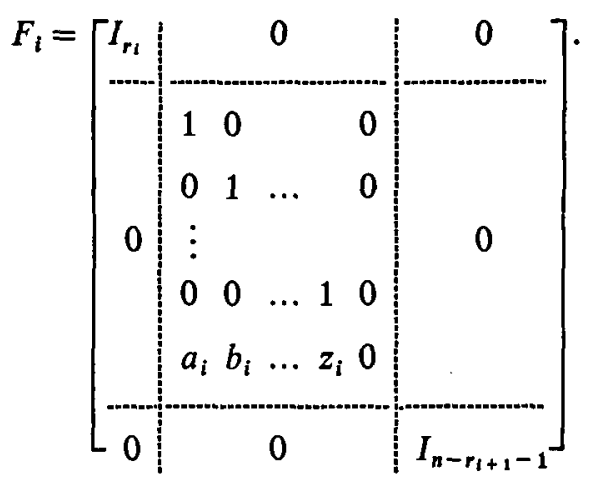

Then a calculation shows that, for each $i, F_{i}$ is idempotent and that, if

$$
B=N F_{1} F_{2} \ldots F_{k-1},
$$

then $B$ coincides with $A$ except in the last row, the last row of $B$ being zero.

Now let

$$
G=\left[\begin{array}{c:c:ccccc}
I_{r k} & \multicolumn{7}{|c}{0} & 0 & & \\
\hdashline & 0 & 0 & \ldots & 0 & 0 \\
0 & 1 & 0 & \ldots & 0 & 0 \\
\vdots & & & & & \\
0 & 0 & 0 & \ldots & 1 & 0 \\
& b_{k} & c_{k} & d_{k} & \ldots & z_{k} & 0
\end{array}\right]
$$

Then, since $a_{k}=0, G B=A$ and $G$ is an idempotent. Therefore $A$ is the product

$$
G E_{n-1} E_{n-2} \ldots E_{1} E_{0} F_{1} F_{2} \ldots F_{k-1}
$$

and the theorem is proved. 


\section{REFERENCES}

1. J. M. Howie, The subsemigroup generated by the idempotents of a full transformation semigroup, J. London Math. Soc. 41 (1966), 707-716.

2. B. L. van der Waerden, Modern algebra, Vol. II (New York, 1950).

UNIVERSITY OF GLASGOW

GLASGOW, W.2

Present AdDRess: King's College, LoNdon, W.C.2 